\title{
Antiretroviral medication for preventing HIV infection in nonoccupational settings
}

\author{
Isaac I. Bogoch MD, Eileen P. Scully MD PhD, Kimon C. Zachary MD
}

$\mathrm{H}$ IV is primarily transmitted through unprotected sexual encounters, contaminated needles and from mother to child during the peripartum period or while breastfeeding. Several evidence-based approaches are used for preventing HIV transmission that can be broadly categorized into pharmacologic and nonpharmacologic strategies. Here, we review the evidence and clinical practice for pharmacologic strategies employed to prevent HIV transmission among people who have been exposed to the virus or who are at high risk for acquiring the infection in nonoccupational settings. These strategies include postexposure prophylaxis, preexposure prophylaxis and starting antiretroviral medications early.

Data concerning postexposure prophylaxis are limited, and clinical practice is primarily guided by case-control or observational studies. Recent large randomized clinical trials form the evidence base for pre-exposure prophylaxis and early intiation of antiretroviral therapy (Box 1).

There have been several recent advances in research on this topic, and we encourage readers to view cited articles and guidelines for definitive management strategies. This review does not discuss the prevention of mother-to-child transmission of the virus or exposure to HIV in occupational settings. Furthermore, although nonpharmacologic strategies such as counselling on safer sexual practices, condom use, circumcision and clean needle exchanges are essential in preventing HIV transmission and should be inte-

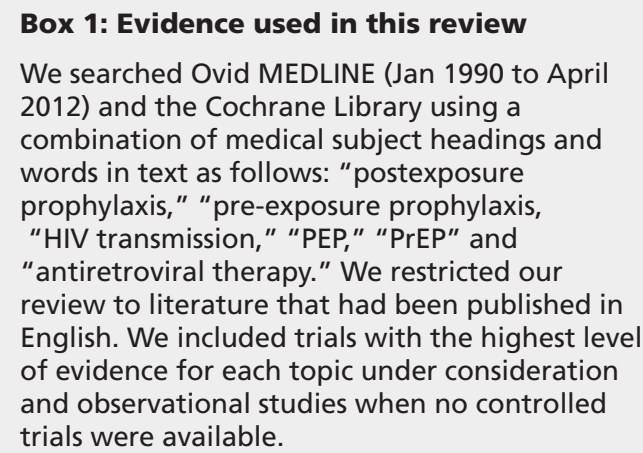

grated with other efforts to control the spread of the virus, the focus of this article is on pharmacologic prevention methods.

\section{Postexposure prophylaxis}

Patients often present to emergency departments or primary care providers after possible exposure to HIV. Several issues may be addressed at the first consultation, including determining whether an exposure has occurred and, if so, the risk of transmission. If an exposure is likely to have occurred, there should be a discussion about postexposure prophylaxis.

\section{Has an HIV exposure occurred?}

A very detailed history is essential in determining whether a person has been exposed to HIV. An exposure occurs when infected body fluids or mucosa from a person with HIV come into contact with the mucosa, blood stream or broken skin of someone else. Infectious fluids include blood, genital secretions, and amniotic, cerebrospinal, pleural, pericardial, peritoneal and synovial fluids. Urine, saliva, sweat, emesis and feces are not considered infectious unless they contain blood.

\section{What is the risk of HIV transmission?}

The risk of HIV transmission differs with the type of exposure; however, it is relatively low for individual exposures. Data on HIV transmission are estimated from observational trials involving couples in which only one partner has HIV infection, ${ }^{1}$ patients with nonoccupational exposure to needles, ${ }^{2}$ recipients of blood transfusions from HIV-positive sources ${ }^{3}$ and estimates from sexual contacts (insertive or receptive oral, penile, vagi-
Competing interests: None declared.

This article has been peer reviewed.

Correspondence to: Dr. Isaac I. Bogoch, Isaac.bogoch@utoronto.ca

CMAJ 2012. DOI:10.1503 /cmaj.120267 
nal and anal contacts). ${ }^{1,4,5}$ Estimated risks of transmission for each type of exposure, per 10000 exposures, are summarized in Table $1 .^{6}$

\section{What is the evidence for nonoccupational postexposure prophylaxis?}

The evidence for nonoccupational postexposure prophylaxis is limited and restricted to one casecontrol study and several observational cohort studies.

The earliest evidence for nonoccupational postexposure prophylaxis was extrapolated from a retrospective case-control study in 1997 that enrolled health care workers (33 cases, 665 controls) with occupational exposures to HIV through percutaneous injuries. ${ }^{7}$ Cardo and coauthors reported an $81 \%$ reduction in HIV seroconversion using zidovudine monotherapy (odds ratio [OR] $0.19,95 \%$ confidence interval [CI] 0.06-0.52). ${ }^{7}$ Risk factors for HIV transmission in this study included deep injury with a contaminated device (OR 15, 95\% CI 6-41), visible blood on the penetrating device (OR 6.2, 95\% CI 2.2-21), a device that had recently been in the artery or vein of a patient with HIV (OR 4.3, 95\% CI 1.7-12), or exposure to a patient who died from AIDS within two months (OR 5.6, 95\% CI 0.06-0.52). ${ }^{7}$ This study prompted widescale adoption of postexposure prophylaxis as a strategy for preventing HIV transmission in both occupational and nonoccupational settings.

Several observational cohort studies done since the study by Cardo and coauthors have assessed HIV seroconversion after postexposure prophylaxis among people with nonoccupational exposures to the virus. A study from San Francisco showed seven seroconversions among 702 people $(1.0 \%, 95 \%$ CI $0.4-2)$ with high-risk

Table 1: Estimated risk of HIV transmission by type of exposure to an HIVpositive source $^{6}$

\begin{tabular}{|lc|}
\hline Type of exposure* & $\begin{array}{c}\text { Estimated risk of HIV transmission } \\
\text { per } 10000 \text { exposures }\end{array}$ \\
\hline Blood transfusion & 9000 \\
\hline $\begin{array}{l}\text { Intravenous drug use with shared } \\
\text { needle }\end{array}$ & 67 \\
\hline Receptive anal intercourse & 50 \\
\hline Percutaneous needle stick & 30 \\
\hline Receptive penile-vaginal intercourse & 10 \\
\hline Insertive anal intercourse & 6.5 \\
\hline Insertive penile-vaginal intercourse & 5 \\
\hline Receptive oral intercourset & 1 \\
\hline Insertive oral intercourse $t$ & 0.5 \\
\hline *Assuming no condom use. \\
tRefers to oral intercourse performed on a man.
\end{tabular}

exposures (most commonly unprotected anal intercourse between men) who came to a followup visit at 12 weeks. ${ }^{8}$ All of the participants had been given a two-drug antiretroviral regimen that began within 72 hours of their exposure. All those who showed seroconversion were men presenting after receptive anal intercourse.

A study from Boston using historical controls showed that substantially more people completed 28-day postexposure prophylaxis regimens containing tenofovir rather than zidovudine, mostly because of tenofovir's favourable adverse-effect profile. ${ }^{9}$ No seroconversions were reported in this study, and tenofovir is now widely used in postexposure prophylaxis regimens.

The data from these studies suggest that postexposure prophylaxis is effective; however, no randomized prospective data exists, and it is unlikely that such a trial will ever be done.

\section{When should postexposure prophylaxis start?}

Postexposure prophylaxis should be started within 72 hours of exposure to HIV (but as soon as possible) and continued for 28 days. ${ }^{6}$ People with high-risk exposures, such as unprotected sex or sharing needles with a person known to have HIV, should be offered postexposure prophylaxis. Current guidelines endorse case-bycase postexposure prophylaxis for a broad range of exposures including low-risk situations such as insertive oral-genital contact with a person of unknown HIV status (Table 1). ${ }^{6}$ Given the paucity of prospective data, the choice to offer postexposure prophylaxis in very low, but nonzerorisk situations may be at the discretion of the provider with appropriate patient counseling on the risks of HIV transmission. Evidence for quickly starting prophylaxis and a four-week duration of therapy stem from macaque models of transmission, in which starting prophylaxis later and shorter durations of therapy resulted in higher rates of HIV seroconversion. ${ }^{10}$ Starting prophylaxis more than 72 hours after an exposure is not recommended, although some practitioners may still do so at their own discretion.

Testing of the person to whom the patient was exposed should be sought whenever possible, but it is often very difficult to do so. All patients should undergo baseline screening for HIV before starting prophylactic treatment. The regimen can be stopped if the source patient is available, consents to HIV testing, has a negative test result and is not suspected to have acute HIV infection. ${ }^{11}$ Symptoms of acute infection include a mononucleosis-like syndrome consisting of fever and malaise, and sometimes pharyngitis, rash and peripheral lymphadenopathy; however, 
the absence of such symptoms does not rule out acute infection. ${ }^{11}$ Diagnosis of acute HIV is difficult because serologic tests are not typically positive until at least 22 days after infection and may remain negative for as many as three or, less commonly, six months. Techniques to amplify HIV RNA may be used to detect infection earlier (16-18 d); however, enzyme-linked immunosorbent assays and western blots should be used to confirm infection if nucleic acid testing is performed. ${ }^{11}$

\section{Which drugs should be used in postexposure prophylaxis?}

Current guidelines recommend two or three antiretroviral medications based on the risk of exposure, with three drug regimens recommended for people with high-risk exposures, such as receptive anal intercourse with a person who is known to have HIV, or other mucosal exposures to people who have detectable viral loads, with higher viral loads portending a greater risk of transmission. ${ }^{6}$

Theoretically, all classes of HIV medications will be effective in preventing HIV transmission, but certain medications are not commonly used because of adverse effects, tolerability and drug interactions. These agents include nevirapine, given the high risk of severe skin reactions and hepatotoxicity in people with normal CD4 T cell counts, and abacavir, given the 5\% risk of potentially severe hypersensitivity. Efavirenz is not usually used because of the neuropsychiatric adverse effects that are common during the first two to four weeks of therapy. ${ }^{9}$

Commonly used two-drug regimens include tenofovir plus emtricitabine, which can be given together as a once daily combination pill with few adverse effects (Table 2). ${ }^{9}$ If a third drug is added, protease inhibitors, such as lopinavir, atazanavir or darunavir, all boosted with ritonavir, are commonly used. Protease inhibitors have many drug interactions, particularly with agents metabolized by cytochrome P450 3A4, often causing gastrointestinal adverse effects (particularly with lopinavir). ${ }^{12}$ More recently, the integrase inhibitor raltegravir has been used when a third drug is added, because of its superior tolerability and few drug interactions. ${ }^{12}$

\section{How are patients followed-up?}

Once a postexposure prophylaxis regimen is started, patients are typically followed-up with blood tests after 2 weeks to assess for renal or liver toxicity, and with HIV screening at baseline, 6 weeks, 12 weeks and 6 months. ${ }^{6}$ Patients are counselled to have only protected intercourse (oral, vaginal and anal) at least until a six-month HIV screening test is confirmed as having a negative result.

Visits to clinics regarding postexposure prophylaxis are often "teachable moments" and are excellent opportunities for counselling on safer sexual practices or connecting patients with appropriate social or financial resources.

\section{Are there additional considerations?}

Patients should be concurrently evaluated for other possible health concerns, including pregnancy or exposure to tetanus, hepatitis B and C, and other sexually transmitted infections such as chlamydia, gonorrhea, trichomonas and genital herpes. People who have been sexually assaulted should be offered presumptive treatment for chlamydia, gonorrhea and trichomonas, with women also being offered emergency contraception. ${ }^{6}$ Vaccination against hepatitis B (and in certain high-risk cases, with hepatitis B immune globulin) should be given to people who are not immune to this virus. ${ }^{6}$

\section{Pre-exposure prophylaxis}

\section{What is pre-exposure prophylaxis?}

Pre-exposure prophylaxis targets high-risk populations with the goal of preventing HIV infection using intermittent or continuous antiretroviral therapy. Unlike postexposure prophylaxis, preexposure prophylaxis requires the at-risk person to act before possible exposure to the virus. For this reason, its application is limited to only a few populations. All pre-exposure prophylaxis interventions should be considered one part of a more comprehensive plan for preventing the spread of HIV infection, including standard counselling on safer sexual practices and condom use, testing for and treating other sexually transmitted infections and, in select circumstances, male circumcision and needle exchange programs. ${ }^{13}$

Table 2: Commonly used and recommended regimens for HIV prophylaxis ${ }^{6,9,12}$ Regimen Duration, d

\section{Postexposure prophylaxis}

Tenofovir (300 mg) and emtricitabine $(200 \mathrm{mg})$, once daily* $\dagger$ 28 Lamivudine $(150 \mathrm{mg})$ and zidovudine $(300 \mathrm{mg})$, twice daily* $†$ 28 Pre-exposure prophylaxis

Tenofovir (300 mg) and emtricitabine $(200 \mathrm{mg})$, once daily Indefinite

*A combination tablet is available for these medications.

tIf a third agent is required, consider either raltegravir ( $400 \mathrm{mg}$, twice daily), lopinavir (400 $\mathrm{mg}$ )/ritonavir $(100 \mathrm{mg}$ ) combination tablet (two tablets, twice daily), darunavir $(800 \mathrm{mg})$ plus ritonavir $(100 \mathrm{mg})$ once daily, or atazanavir $(300 \mathrm{mg})$ plus ritonavir $(100 \mathrm{mg})$ once daily. $\ddagger$ Consider use in high-risk patients after consulting guidelines. ${ }^{13}$ 


\section{What is the evidence for pre-exposure prophylaxis?}

There have been several recent large trials for preexposure prophylaxis, some with marked success in preventing HIV transmission. The first study to show the efficacy of pre-exposure prophylaxis was the CAPRISA (Centre for the AIDS Program of Research in South Africa) 004 study, which investigated a topical vaginal microbicide for the prevention of HIV transmission in a randomized, double-blind, placebo-controlled trial. ${ }^{14}$ Almost 900 women from a region of high HIV prevalence were instructed to apply the gel containing tenofovir or placebo no more than 12 hours before a sexual encounter and again within 12 hours after sex. The overall effectiveness in reducing rates of HIV infection was 39\%, with an incidence of 5.6 infections per 100 woman-years (95\% CI 4-7.7) in the treatment group compared with 9.1 per 100 woman-years (95\% CI 6.9-11.7) in the placebo group. Higher levels of protection were seen among women with better adherence to treatment. For example, among women with $80 \%$ adherence or better, the incidence of HIV in the treatment group was 4.2 per 100 woman-years $(95 \% \mathrm{CI}$ 2.1-7.6) compared with 9.3 per 100 woman-years in the placebo arm (95\% CI 6-13.7), for a $54 \%$ reduction in the incidence of HIV infection with the use of tenofovir gel.

Pre-exposure prophylaxis was also found to be efficacious in the Preexposure Prophylaxis Initiative study, a large, randomized, placebocontrolled, international trial of a continuous prevention strategy consisting of a once daily pill containing emtricitabine and tenofovir. ${ }^{15}$ This study enrolled 2499 men (and transgender women) who have sex with men, with high-risk sexual behaviours including an average of 18 sexual partners in the three months before presentation. About onehalf of the participants reported having unprotected anal sex during that time period. In addition to receiving either daily emtricitabine/tenofovir or placebo, all participants were counselled on safer sexual practices and underwent testing and treatment for coincident sexually transmitted infections. The cohort was followed for 3324 personyears, and the overall results yielded a $44 \%$ relative reduction (95\% CI 15-63) in rates of HIV acquisition among men given emtricitabine/ tenofovir, with 36 HIV seroconversions occurring in the treatment group; 64 seroconversions occurred in the placebo group. As in the CAPRISA study, ${ }^{14}$ protection strongly correlated with adherence to the drug regimen. Participants who were randomized to the emtricitabine/tenofovir group and took their pills more than $90 \%$ of the time showed a $73 \%$ relative reduction $(95 \%$ CI 41-88) in the incidence of HIV.
A third large trial from 2011 showed less promising results. The FemPrEP study, sponsored by Family Health International, ${ }^{16}$ was a randomized, placebo-controlled trial of oral emtricitabine/tenofovir involving African women that showed an equivalent number of infections in both the treatment and placebo arms. The study was stopped prematurely, because it was unlikely to show any effectiveness of treatment. The reasons for the study's failure are currently unclear; however, further analyses may help to explain these results.

\section{What are the controversies surrounding pre-exposure prophylaxis?}

Pre-exposure prophylaxis offers promising results in terms of preventing the spread of HIV, but controversies exist regarding appropriate recipients, implementation and costs. ${ }^{17}$ Some concerns involve the possibility for increased risk-taking behaviour, the risk of viral resistance to antiretroviral drugs when infection does occur while on prophylaxis, the potential for longterm adverse effects and the cost of the therapy.

Tenofovir has been associated with reductions in bone density and, sometimes, with dysfunction of the proximal renal convoluted tubules. In addition, emtricitabine and tenofovir have activity against hepatitis $\mathrm{B}$, and abrupt withdrawal of these drugs has been associated with flares of hepatitis. ${ }^{17}$ For this reason, interim guidance from the Centers for Disease Control and Prevention (CDC) recommend patients undergo baseline testing for hepatitis $B$, and vaccination when appropriate, before starting treatment. ${ }^{13}$ The Preexposure Prophylaxis Initiative ${ }^{15}$ incorporated substantial prevention counselling and frequent HIV testing, presenting further challenges to its broad implementation, but did not show any increases in risk-taking behaviours among its participants.

Currently, interim guidance from the CDC suggests that pre-exposure prophylaxis be considered for men at high risk of exposure who have sex with other men, with the first evaluation including documenting ongoing high-risk behaviour, baseline renal function, screening for HIV, and testing and treatment or vaccination for hepatitis B and concurrent sexually transmitted infections (Table 2). ${ }^{13}$

\section{Starting treatment early after infection}

Possibly the most effective way to prevent the spread of HIV is by providing treatment to people who already have the virus. Antiretroviral therapy reduces the amount of virus in blood and 
genital secretions, thereby reducing the possibility of further transmission. Because HIV is a chronic infection, treatment currently involves lifelong suppression of the virus with adherence to daily antiretroviral medications. ${ }^{18}$ Mathematical models predict that the incidence of HIV can be substantially reduced with universal testing and immediate treatment. ${ }^{19}$

A recent international randomized trial involved couples in whom only one partner had HIV. The partners with HIV were randomized to receive either early treatment (at the time of enrollment) or delayed treatment (when the CD4 count dropped below 250 cells $/ \mathrm{mL}$ or at the onset of an AIDS-related illness). Among the 1763 couples enrolled, there were 28 HIV transmissions virologically linked to the partner with HIV, and only one of these transmissions oc curred in the early treatment group (hazard ratio $0.04,95 \%$ CI $0.01-0.88) .^{20}$

The early treatment of HIV, which is the standard in most developed countries, may have health benefits for the people living with the virus while reducing transmission at a population level.

\section{Conclusion}

Several pharmacologic strategies exist to prevent HIV infection in people who are at risk of exposure to the virus. Although postexposure prophylaxis has a long history of success, newer methods such as pre-exposure prophylaxis and earlier treatment in the course of infection ("treatment as prevention") are being implemented with some success. Whereas pre-exposure prophylaxis may be reserved for people with the highest risk of exposure, the trend of treating HIV at higher CD4 $\mathrm{T}$ cell counts earlier in infection will likely show the most promise as a pharmacologic strategy for preventing transmission of the virus.

There are several unanswered questions about pre-exposure prophylaxis, including which groups would derive the most benefit (e.g., men who have sex with men, people who use intravenous drugs), the efficacy of continuous antiretroviral prophylaxis versus intermittent prophylaxis, and the possibility of drug resistance developing while receiving prophylactic therapy. Several ongoing trials are seeking to answer these questions. In addition, large-scale international trials are underway to further elucidate the effectiveness of early treatment as a preventive strategy at the individual and community levels.

\section{References}

1. Varghese B, Maher JE, Peterman TA, et al. Reducing the risk of sexual HIV transmission: quantifying the per-act risk for HIV on the basis of choice of partner, sex act, and condom use. Sex Transm Dis 2002;29:38-43.
2. Kaplan EH, Heimer R. HIV incidence among New Haven needle exchange participants: updated estimates from syringe tracking and testing data. J Acquir Immune Defic Syndr Hum Retrovirol 1995; 10:175-6.

3. Donegan E, Stuart M, Niland JC, et al. Infection with human immunodeficiency virus type 1 (HIV-1) among recipients of antibody-positive blood donations. Ann Intern Med 1990;113: 733-9.

4. European Study Group on Heterosexual Transmission of HIV. Comparison of female to male and male to female transmission of HIV in 563 stable couples. BMJ 1992;304:809-13.

5. Leynaert B, Downs AM, De Vincenzi I; European Study Group on Heterosexual Transmission of HIV. Heterosexual transmission of HIV: variability of infectivity throughout the course of infection. Am J Epidemiol 1998;148:88-96.

6. Smith DK, Grohskopf LA, Black RJ, et al. Antiretroviral postexposure prophylaxis after sexual, injection-drug use, or other nonoccupational exposure to HIV in the United States: recommendations from the US Department of Health and Human Services. MMWR Recomm Rep 2005;54(RR-2):1-20.

7. Cardo DM, Culver DH, Ciesielski CA, et al. A case-control study of HIV seroconversion in health care workers after percutaneous exposure. Centers for Disease Control and Prevention Needlestick Surveillance Group. N Engl J Med 1997;337:1485-90.

8. Roland ME, Neilands TB, Krone MR, et al. Seroconversion following nonoccupational postexposure prophylaxis against HIV. Clin Infect Dis 2005;41:1507-13.

9. Mayer KH, Mimiaga MJ, Cohen D, et al. Tenofovir DF plus lamivudine or emtricitabine for nonoccupational postexposure prophylaxis (NPEP) in a Boston Community Health Center. $J$ Acquir Immune Defic Syndr 2008;47:494-9.

10. Tsai CC, Emau P, Follis KE, et al. Effectiveness of postinoculation (R)-9-(2 phosphonylmethoxypropyl) adenine treatment for prevention of persistent simian immunodeficiency virus SIVmne infection depends critically on timing of initiation and duration of treatment. J Virol 1998;72:4265-73.

11. Kahn JO, Walker BD. Acute human immunodeficiency virus type 1 infection. N Engl J Med 1998;339:33-9.

12. Mayer KH, Mimiaga MJ, Gelman M, et al. Raltegravir, tenofovir DF, and emtricitabine for post-exposure prophylaxis to prevent the sexual transmission of HIV: safety, tolerability and adherence. J Acquir Immune Defic Syndr 2012;59:354-9.

13. Abdool Karim Q, Abdool Karim SS, Frohlich JA, et al. Effectiveness and safety of tenofovir gel, an antiretroviral microbicide, for the prevention of HIV infection in women. Science 2010;329:1168-74.

14. Grant RM, Lama JR, Anderson PL, et al. Preexposure chemoprophylaxis for HIV prevention in men who have sex with men. N Engl J Med 2010;363:2587-99.

15. FEM-PrEP (Truvada $®)$ : Study to assess the role of Truvada $®$ in preventing HIV acquisition in women [clinical trial]. Bethesda (MD): US National Institutes of Health. Available: http://clinicaltrials.gov/ct2/show/NCT00625404 (accessed 2011 Nov. 9).

16. Mayer KH. Antiretrovirals for HIV prevention: translating promise into praxis. Lancet 2011;378:206-8.

17. Interim guidance: preexposure prophylaxis for the prevention of HIV infection in men who have sex with men. MMWR Morb Mortal Wkly Rep 2011;60:65-8.

18. Thompson MA, Aberg JA, Cahn P, et al. Antiretroviral treatment of adult HIV infection: 2010 recommendations of the International AIDS Society-USA panel. JAMA 2010;304:321-33.

19. Granich RM, Gilks CF, Dye C, et al. Universal voluntary HIV testing with immediate antiretroviral therapy as a strategy for elimination of HIV transmission: a mathematical model. Lancet 2009;373:48-57.

20. Cohen MS, Chen YQ, McCauley M, et al. Prevention of HIV-1 infection with early antiretroviral therapy. N Engl J Med 2011; 365:493-505.

Affiliations: From the Division of Infectious Diseases (Bogoch), Sunnybrook Health Sciences Centre, Toronto, Ont.; the Division of Infectious Diseases (Bogoch, Zachary), Massachusetts General Hospital; the Division of Infectious Diseases (Scully), Brigham and Women's Hospital; and Harvard Medical School (Bogoch, Scully, Zachary), Boston, Mass.

Contributors: All of the authors substantially contributed to the conception, design, drafting and revising of the manuscript. All of the authors approved the final version submitted for publication. 